\title{
Genetic correlations between pain phenotypes and depression and neuroticism
}

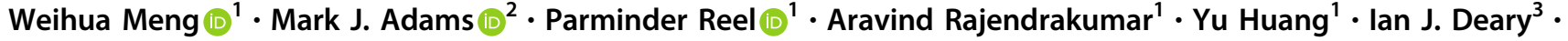 \\ Colin N. A. Palmer ${ }^{1} \cdot$ Andrew M. McIntosh $\mathbb{B}^{2,3} \cdot$ Blair H. Smith $\mathbb{D}^{1}$
}

Received: 22 May 2019 / Revised: 23 September 2019 / Accepted: 27 September 2019 / Published online: 29 October 2019

(c) The Author(s) 2019. This article is published with open access

\begin{abstract}
Correlations between pain phenotypes and psychiatric traits such as depression and the personality trait of neuroticism are not fully understood. In this study, we estimated the genetic correlations of eight pain phenotypes (defined by the UK Biobank, $n=151,922-226,683$ ) with depressive symptoms, major depressive disorders and neuroticism using the the crosstrait linkage disequilibrium score regression (LDSC) method integrated in the LD Hub. We also used the LDSC software to calculate the genetic correlations among pain phenotypes. All pain phenotypes, except hip pain and knee pain, had significant and positive genetic correlations with depressive symptoms, major depressive disorders and neuroticism. All pain phenotypes were heritable, with pain all over the body showing the highest heritability $\left(h^{2}=0.31\right.$, standard error $\left.=0.072\right)$. Many pain phenotypes had positive and significant genetic correlations with each other indicating shared genetic mechanisms. Our results suggest that pain, neuroticism and depression share partially overlapping genetic risk factors.
\end{abstract}

\section{Introduction}

Pain is a global public health priority. In the Global Burden of Diseases Study 2017, low back and headache disorders were the leading cause of years lived with disability (YLDs) worldwide, with neck pain also ranked among the top ten causes [1]. Some other leading causes of YLDs such as musculoskeletal disorders and diabetes are also highly likely to feature pain as a prominent symptom. It was estimated that $20 \%$ of adults suffer from pain globally and that $10 \%$

These authors contributed equally: Weihua Meng, Mark J. Adams

Supplementary information The online version of this article (https:// doi.org/10.1038/s41431-019-0530-2) contains supplementary material, which is available to authorized users.

$\triangle$ Weihua Meng

w.meng@dundee.ac.uk

1 Division of Population Health and Genomics, School of Medicine, University of Dundee, Dundee DD2 4BF, UK

2 Division of Psychiatry, Edinburgh Medical School, University of Edinburgh, Edinburgh EH10 5HF, UK

3 Centre for Cognitive Ageing and Cognitive Epidemiology, Department of Psychology, University of Edinburgh, Edinburgh EH8 9JZ, UK adults are diagnosed with chronic pain each year [2]. Chronic pain, i.e. pain that has persisted beyond normal tissue healing time (usually taken as 3 months) can arise from many causes, but is often idiopathic or difficult to classify pathophysiologically [3]. It is recognized to have significant genetic contributions to its development [4]. Improving our understanding of the genetic contributions to the experience of pain could help our understanding of its aetiology and prevention. Although some genetic studies have focused on pain in specific body sites and have suggested possible genetic variants associated with pain phenotypes [5], the overall understanding of the genetics of pain remain unclear. Current limitations in our knowledge include: 1 . the extent to which pain as a phenotype is determined by additive genetic components mainly represented by single nucleotide polymorphisms (SNPs); 2. whether the genetic mechanisms of pain in different body sites or in different disorders are similar or different; and 3. whether the genetic connections between pain phenotypes and other common comorbidities are similar or different. Addressing these questions brings further challenges when the severity and the frequency of pain are taken into account.

Both depression and neuroticism (a personality trait) are common comorbidities of pain [6,7]. Depression was ranked as the third most important cause of disability worldwide and neuroticism was also with significant global 
health impacts [1, 8]. Many pain phenotypes have been shown to be associated with depression and neuroticism in epidemiological studies [9-18]. This epidemiological coexistence could arise in part because of shared genetic factors $[19,20]$. Understanding the genetic correlations between pain, depression and neuroticism may help to elucidate their degree of shared genetic architecture and provide a framework for future causal inference [21]. It has been proposed that some pain phenotypes (such as migraine, back pain) and depression or neuroticism share common genetic components [17, 22-24]. However, to the best of our knowledge, the genetic correlations between multiple pain phenotypes in different body sites, and those between pain phenotypes and depressive symptoms, major depressive disorders and neuroticism, have not been reported systematically. Genetic correlation, which refers to the proportion of variance that two traits share due to genetic causes, can contribute to the validation of genomewide association study (GWAS) results, prediction of traits, and explaining the underlying aetiologies.

In order to identify genetic correlations between pain phenotypes and depression and the personality trait of neuroticism, as well as the genetic correlations among pain phenotypes, we generated GWAS summary statistics on eight pain phenotypes in different body sites based on the UK Biobank cohort and adapted the cross-trait linkage disequilibrium score regression (LDSC) method through the LD hub (http://ldsc.broadinstitute.org/ldhub/) and the LDSC software (https://github.com/bulik/ldsc) [25].

\section{Materials and methods}

\section{Participants}

Over 500,000 people aged between 40 and 69 years were recruited by the UK Biobank cohort in 2006-2010 across England, Scotland and Wales. A detailed clinical, demographic, and lifestyle questionnaire was completed by all participants. Biological samples (blood, urine and saliva) were also provided for future analysis. Further information on the UK Biobank cohort can be found at www.ukbioba nk.ac.uk. Ethical approval was granted by the National Health Service National Research Ethics Service (reference 11/NW/0382). The current study was conducted under approved UK Biobank data application number 4844.

DNA extraction and quality control (QC) were standardized and the detailed method can be found at http://www. ukbiobank.ac.uk/wp-content/uploads/2014/04/DNA-Extra ction-at-UK-Biobank-October-2014.pdf. Genotyping was obtained from the bespoke Affymetrix UK Biobank chips. The Wellcome Trust Centre for Human Genetics at Oxford University was in charge of standard QC procedures for genotyping results. The detailed QC steps can be found at http://biobank.ctsu.ox.ac.uk/crystal/refer.cgi?id $=155580$.

In March 2018, The UK Biobank released an updated version of the genetic information (including directly genotyped genotypes and imputed genotypes) of 501,708 samples to all approved researchers. The detailed QC steps of imputation were described by Bycroft et al. [26].

\section{Definitions of pain phenotypes}

We used a specific pain-related questionnaire adapted by the UK Biobank, which included the question: 'In the last month have you experienced any of the following that interfered with your usual activities?'. The options were: 1 . headache; 2 . facial pain; 3 . neck or shoulder pain; 4. back pain; 5. stomach or abdominal pain; 6. hip pain; 7. knee pain; 8. pain all over the body; 9. none of the above; 10 . prefer not to say. More than one option could be selected. (UK Biobank Questionnaire field ID: 6159) (http://biobank. ndph.ox.ac.uk/showcase/refer.cgi?id=100527).

For each pain phenotype, cases were defined as those who selected the specific pain site option for the above question, regardless of whether they had selected other options. For example, headache cases are those who selected the 'headache' option; facial pain cases are those who selected the 'facial pain' option; etc.

For each GWAS analysis, controls were those who selected the 'none of the above' option. Thus we used the same 'no pain' control population for all pain phenotypes in different body sites.

\section{Definitions of depression and neuroticism}

The phenotypes of depression and neuroticism were defined by the psychological cohorts collected by the LD hub [2729]. The original researchers of these cohorts agreed to share the GWAS summary statistics on depression and neuroticism with the LD hub for generating genetic correlations. Therefore, we selected the 'psychiatric diseases' option and the 'personality traits' option in the LD hub to include the depression and neuroticism traits. These traits are: depressive symptoms [27], neuroticism $(\times 2$ studies $)$ [27, 28], major depressive disorder [29]. However, for neuroticism, we only chose the version used by Okbay et al. [27], as it is a GWAS meta-analysis publication, the results of which included the results from van den Berg et al. [28].

\section{Statistical analysis}

\section{Generating the heritabilities of all pain phenotypes}

In this study, genome-wide complex trait analysis was used to calculate narrow-sense SNP-based heritabilities on a 
liability scale based on the genomic-relatedness-based restricted maximum-likelihood approach [30].

\section{Generating GWAS summary statistics of all pain phenotypes}

In this study, genotype data were analysed in BGENIE (https://jmarchini.org/bgenie/), as recommended by UK Biobank. Routine QC steps included: removing SNPs with INFO scores $<0.1$, SNPs with minor allele frequency $<0.5 \%$, or SNPs that failed Hardy-Weinberg tests $P<10^{-6}$. SNPs on the $\mathrm{X}$ and $\mathrm{Y}$ chromosomes and mitochondrial SNPs were also removed. We further removed those whose ancestry was not white British based on principal component analysis, those who were related at least another participant in the cohort (a cut-off value of 0.044 in the generation of the genetic relationship matrix) and those who failed QC. Association tests based on standard Frequentist association were performed using BGENIE adjusting for age, sex, body mass index (BMI), nine population principal components, genotyping arrays, and assessment centres.

\section{Generating genetic correlations between pain phenotypes and depression and neuroticism by the LD hub}

The LD hub has gathered 235 published GWAS summary statistics of different disorders worldwide. Those GWAS summary statistics were compared against researcheruploaded GWAS summary statistics of a phenotype of interest to generate genetic correlations between the phenotype and 235 phenotypes [25].

In order to identify genetic correlations between pain phenotypes and the depression and neuroticism traits, we used the cross-trait LDSC method through the LD Hub v1.9.0 [25]. The LD Hub estimates the bivariate genetic correlations of a phenotype with other traits using individual SNP allele effect sizes and the average LD in a region. In this study, those with $P$ values $<0.001(0.05 / 24+28$, eight pain phenotypes and three psychiatric phenotypes, plus 28 pair combinations among pain phenotypes) should be considered significant surviving Bonferroni correction for multiple testing.

\section{Results}

\section{The heritabilities and the GWAS summary statistics of all pain phenotypes}

The specific pain question received 775,252 responses to all options answered by 501,708 UK Biobank participants during the initial assessment visit (2006-2010). Table 1
Table 1 The sample numbers available for GWAS on eight pain phenotypes

\begin{tabular}{lll}
\hline Pain phenotypes & Cases & Controls \\
\hline Headache & 74,761 & 149,312 \\
Facial pain & 2610 & 149,312 \\
Neck or shoulder pain & 53,994 & 149,312 \\
Stomach or abdominal pain & 8217 & 149,312 \\
Back pain & 43,991 & 149,312 \\
Hip pain & 10,116 & 149,312 \\
Knee pain & 22,204 & 149,312 \\
Pain all over body & 5670 & 149,312 \\
\hline
\end{tabular}

summarises the numbers of cases and controls in the GWAS of the eight pain phenotypes. A Supplementary Table 1 presenting the age, sex, and BMI of participants contributing to the eight GWAS and of the non-responders is included. The GWAS summary statistics can be downloaded from the links in the data availability section. The Manhattan plots and Q-Q plots of these GWAS are also included in a Supplementary file.

The narrow-sense SNP heritabilities of each pain phenotype are presented in the Table 2. Pain all over the body had the greatest heritability among all pain phenotypes $\left(h^{2}=0.31\right.$, standard error $\left.(\mathrm{SE})=0.072\right)$. Knee pain has the lowest heritability $\left(h^{2}=0.08, \mathrm{SE}=0.029\right)$.The SNP heritabilities of other pain phenotypes were between 0.11 and 0.24 .

\section{Genetic correlations between pain and depression and neuroticism}

Through the genetic correlation analysis, we identified multiple significant and positive correlations between pain phenotypes and depression and neuroticism. (Table 2, Fig. 1) A supplementary table is provided to show the genetic correlations between pain phenotypes and all available psychiatric and personality traits in the LD hub (Supplementary Table 2).

\section{Depression and eight pain phenotypes}

For depressive symptoms, all pain phenotypes had significant and positive genetic correlations with depression except hip pain and knee pain. The largest genetic correlation occurred with pain all over the body $(\mathrm{rg}=0.69$, $P=1.4 \times 10^{-27}$ ), followed by stomach or abdominal pain $\left(\mathrm{rg}=0.67, P=5.7 \times 10^{-7}\right)$. For hip pain and knee pain, although there were positive genetic correlations with depressive symptoms ( $\mathrm{rg}=0.34$ and 0.12 , correspondingly), the associations did not survive Bonferroni correction $(P=0.03$ and 0.13 , correspondingly). The values 
Table 2 The SNP-based heritabilities $\left(h^{2}\right)$ of eight pain phenotypes from the UK Biobank cohort and their genetic correlations with depressive symptoms, major depression and neuroticism

\begin{tabular}{|c|c|c|c|c|c|c|c|c|}
\hline \multirow[b]{2}{*}{ Pain phenotypes } & \multicolumn{2}{|l|}{ Heritability } & \multicolumn{2}{|c|}{ Depressive symptoms } & \multicolumn{2}{|c|}{ Major depressive disorder } & \multicolumn{2}{|l|}{ Neuroticism } \\
\hline & $h^{2}(\mathrm{SE})$ & $P$ & $\mathrm{rg}$ & $P$ & $\mathrm{rg}$ & $P$ & $\mathrm{rg}$ & $P$ \\
\hline Headache & $0.21(0.015)$ & $3.9 \times 10^{-45}$ & $\mathbf{0 . 5 2}(0.036)$ & $1.6 \times 10^{-46}$ & $\mathbf{0 . 3 9}(0.057)$ & $1.6 \times 10^{-11}$ & $\mathbf{0 . 5 0}(0.028)$ & $2.2 \times 10^{-72}$ \\
\hline Facial pain & $0.24(0.12)$ & 0.036 & $\mathbf{0 . 3 3}(0.091)$ & $2 \times 10^{-4}$ & $0.34(0.137)$ & 0.01 & $\mathbf{0 . 3 0}(0.068)$ & $1.0 \times 10^{-5}$ \\
\hline Neck or shoulder pain & $0.11(0.017)$ & $4.3 \times 10^{-11}$ & $\mathbf{0 . 5 5}(0.048)$ & $3.4 \times 10^{-30}$ & $\mathbf{0 . 4 0}(0.073)$ & $5.8 \times 10^{-8}$ & $\mathbf{0 . 4 4}(0.039)$ & $5.3 \times 10^{-7}$ \\
\hline Stomach or abdominal pain & $0.14(0.050)$ & 0.004 & $\mathbf{0 . 6 7}(0.134)$ & $5.7 \times 10^{-7}$ & $\mathbf{0 . 5 3}(0.15)$ & $5 \times 10^{-4}$ & $\mathbf{0 . 7 0}(0.118)$ & $2.4 \times 10^{-9}$ \\
\hline Back pain & $0.11(0.020)$ & $8.4 \times 10^{-9}$ & $\mathbf{0 . 4 8}(0.063)$ & $1.5 \times 10^{-14}$ & $\mathbf{0 . 3 6}(0.086)$ & $3 \times 10^{-5}$ & $\mathbf{0 . 4 0}(0.054)$ & $1.7 \times 10^{-13}$ \\
\hline Hip pain & $0.12(0.041)$ & 0.005 & $0.34(0.16)$ & 0.03 & $0.04(0.167)$ & 0.80 & $0.27(0.128)$ & 0.04 \\
\hline Knee pain & $0.08(0.029)$ & 0.007 & $0.12(0.083)$ & 0.13 & $-0.07(0.113)$ & 0.53 & $0.18(0.057)$ & 0.002 \\
\hline Pain all over body & $0.31(0.072)$ & $1.3 \times 10^{-5}$ & $\mathbf{0 . 6 9}(0.063)$ & $1.4 \times 10^{-27}$ & $\mathbf{0 . 4 3}(0.095)$ & $5.6 \times 10^{-6}$ & $\mathbf{0 . 4 5}(0.054)$ & $3.4 \times 10^{-17}$ \\
\hline
\end{tabular}

$P$ values $<0.001(0.05 / 24+28)$ were considered as significant for genetic correlations. Those significant rg values were in bold

$S E$ standard error, $r g$ genetic correlation

Fig. 1 The genetic correlations between eight pain phenotypes and depressive symptoms, major depressive disorders and neuroticism. Please note, the genetic correlations between these traits and hip pain and knee pain were not significant $(P>0.001$, Table 2$)$. rg genetic correlation

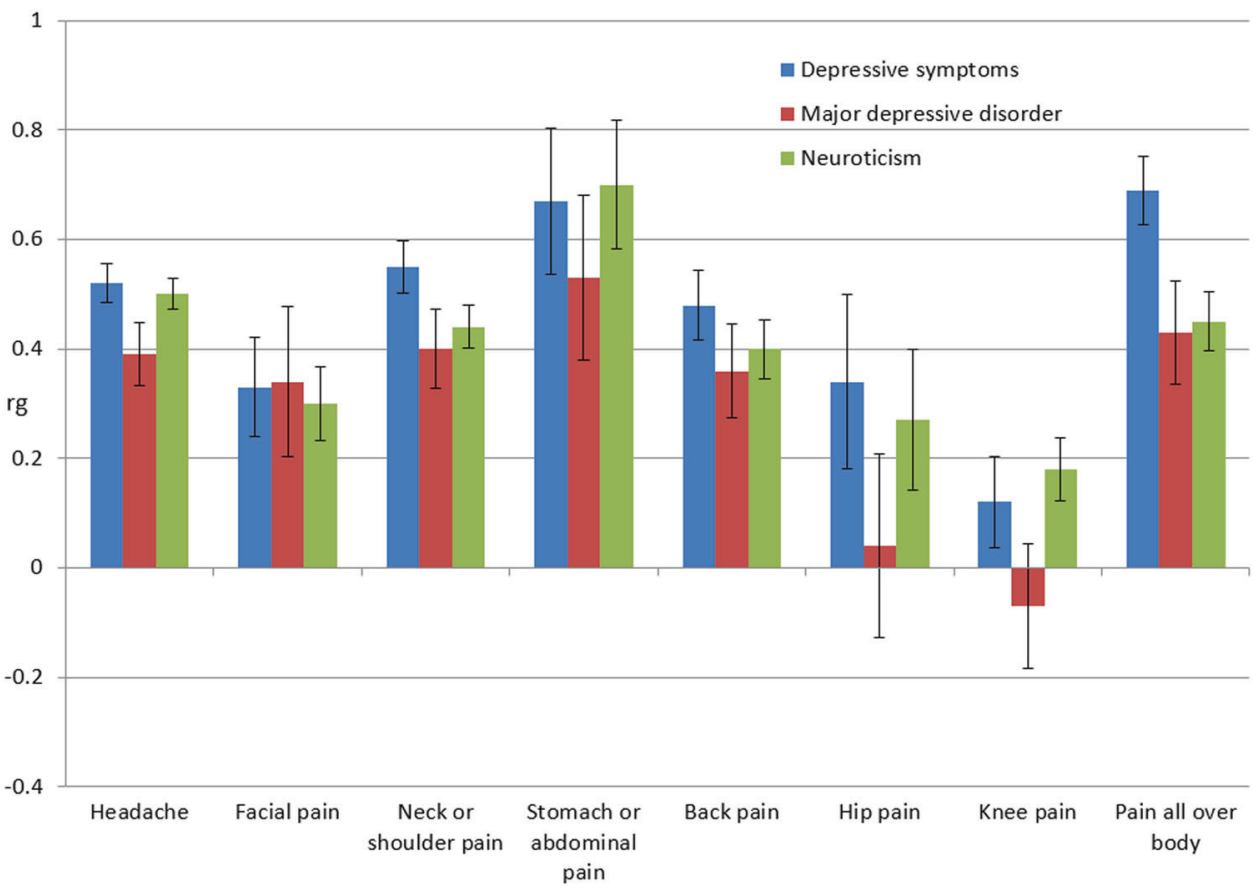

Neuroticism and eight pain phenotypes

of the genetic correlations between other pain phenotypes and depressive symptoms were between $\mathrm{rg}=0.33$ and 0.55 , and all were statistically significant after adjustment for multiple testing.

For major depressive disorder, the genetic correlation results were similar to those for depressive symptoms. The largest genetic correlation was with stomach or abdominal pain ( $\mathrm{rg}=0.53, P=0.0005)$, followed by pain all over the body $\left(\mathrm{rg}=0.43, P=5.6 \times 10^{-6}\right)$. However, the $\mathrm{rg}$ values of hip pain and knee pain were 0.04 and -0.07 , which were also statistically insignificant $(P=0.80$ and 0.53 , correspondingly). The values of the genetic correlations between other pain phenotypes and major depressive disorder were between $\mathrm{rg}=0.34$ and 0.40 , and all were statistically significant.
With neuroticism, stomach or abdominal pain had the largest genetic correlation ( $\left.\mathrm{rg}=0.70, P=2.4 \times 10^{-9}\right)$. Headache followed next with $\mathrm{rg}=0.50$ and $P=2.2 \times 10^{-72}$. All genetic correlations with other pain phenotypes (except hip pain and knee pain) were positive and significant with rg values between 0.30 and 0.50 . For hip pain and knee pain, although there were positive genetic correlations with neuroticism, the correlations were statistically insignificant.

\section{Genetic correlations among pain phenotypes}

Through the LDSC software, we identified multiple significant and positive correlations among pain phenotypes 


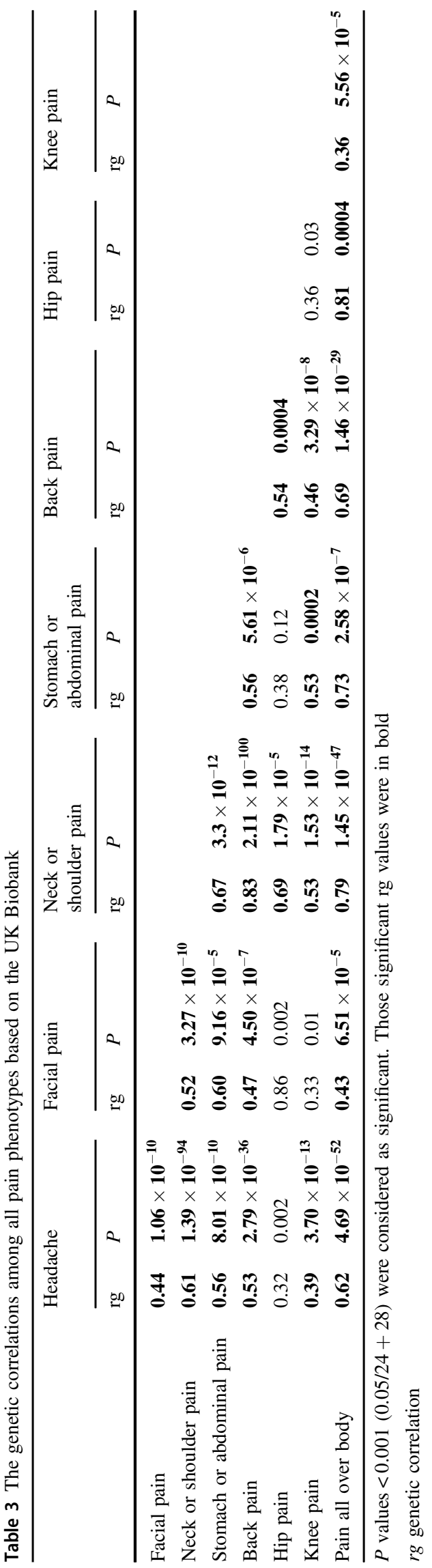

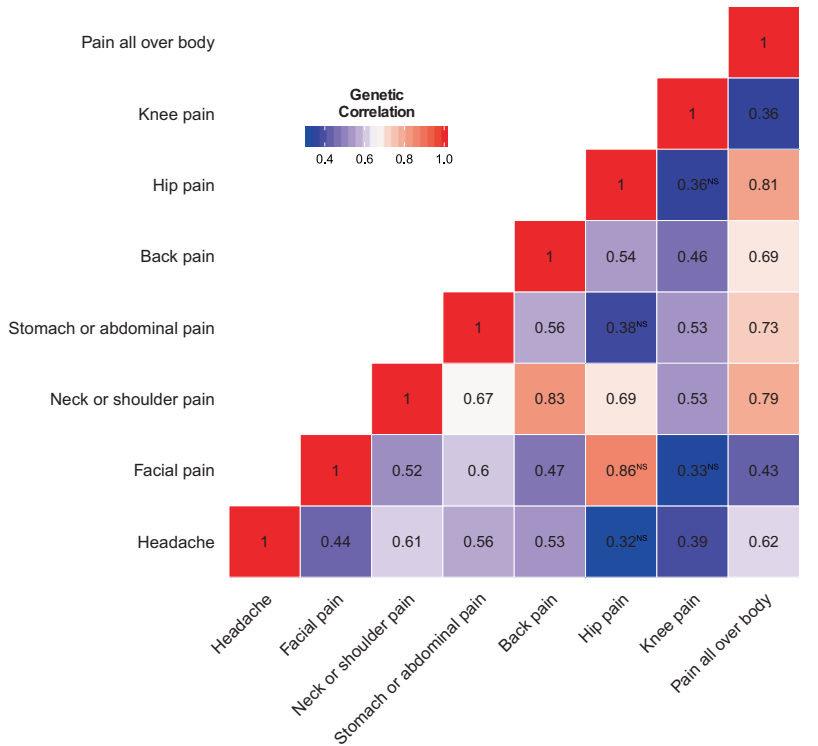

Fig. 2 The heatmap of the genetic correlations among eight pain phenotypes

(Table 3, Fig. 2). The largest positive and significant genetic correlation was between neck or shoulder pain and back pain $\left(\mathrm{rg}=0.83, P=2.11 \times 10^{-100}\right)$, followed by hip pain and pain all over the body ( $\mathrm{rg}=0.81, P=0.0004)$. Neck or shoulder pain had positive and significant genetic correlations with all other pain phenotypes $(0.52<\mathrm{rg}<0.83)$, this was the same for pain all over the body $(0.36<\mathrm{rg}<0.81)$. Among all pain phenotypes, hip pain only had three positive and significant genetic correlations (neck or shoulder pain, back pain and pain all over the body) with other pain phenotypes.

\section{Discussion}

In this study, eight self-reported pain phenotypes, from different sites across the body, were all heritable and showed a broad pattern of partially shared genetic architecture with each other. We also found evidence that pain shared genetic architecture with depressive symptoms, major depressive disorder and neuroticism for most sites across the body. Hip pain and knee pain were the exception, in showing weak and non-significant genetic correlations with depressive symptoms, major depressive disorder and neuroticism.

A significant and positive correlation between a pain phenotype and a psychiatric trait reflects shared genetic architecture. This can reflect pleiotropy, where specific genetic alleles increase risk to both phenotypes, but it can also reflect mediated pleiotropy where there is a directional or causal association between these traits [21]. It is also possible for positive genetic correlations to be generated by 
misclassification of pain as depression, or vice versa [31]. It is important to examine whether there is subgroup heterogeneity amongst individuals with pain and depression that can be explained by variation on the other trait. It is worth mentioning that a genetic correlation between a pain phenotype and a psychiatric trait does not necessarily indicate that a genetic predisposition for a psychiatric trait will increase sensitivity to pain, or vice versa.

The nature of the relationship between pain phenotypes and depression has been uncertain. Epidemiological studies have identified that depression is reported more often by patients reporting pain and also that pain is a risk factor for the future development of depression [28]. Pain was present in around $65 \%$ in depressed patients and up to $85 \%$ of patients with chronic pain were affected by severe depression [32]. Previous studies have reported a genetic correlation between pain and depression of 0.56 (in a sample of twins) and 0.51 (in a family-based samples) [19, 33]. Although the exact mechanisms linking these conditions are not clear, genetic mechanisms are implied through shared biological pathways, such as gene expression in biological networks, the endocannabinoid system, the hypothalamic-pituitary-adrenal axis and inflammatory pathways [34]. Further, imaging studies on brain function support the relationships between pain phenotypes and psychiatric traits. Pain has been associated with functional imaging alterations in brain regions responsible for processing emotional stimuli [35]. Our study answered a specific question about depression: to what extent is each pain phenotype genetically correlated with depression? Our genetic correlation results between headache, facial pain, neck or shoulder pain, stomach or abdominal pain, back pain, pain all over the body and depression are all consistent in direction with known epidemiological associations [914]. This suggests that shared genetic risk factors are likely to partly explain their phenotypic correlations. However, the genetic correlations between hip pain and knee pain and depression were contrary to previous observations that depression and knee pain or hip pain are strongly related [36]. Previous studies have shown that knee pain from osteoarthritis increases a person's risk of developing subsequent depression [37]. A systematic review of the relationship between knee pain and multiple psychiatric traits also found an association between depression and knee pain [38]. While genetic factors may contribute to the pain at different sites, our findings suggest that non-genetic factors may be more important in the co-occurrence of knee or hip pain with depression.

The genetic relationships between pain phenotypes and neuroticism are also of interest and are similar to those between pain and depression. Neuroticism was identified to be a potential risk factor for elevated pain responses in laboratory pain in healthy children, and can likely exacerbate pain responses when coupled with fear of bodily sensations [39]. Neuroticism has also been independently associated with greater pain catastrophizing [7]. Our genetic correlation results were consistent with findings from epidemiological studies of headache [15], neck or shoulder pain [16], back pain [17], and pain all over the body [18]. No previous studies have shown epidemiological data for the relationships between neuroticism and facial pain or stomach or abdominal pain. It was also suggested from our study that there were no significant genetic relationships between knee pain or hip pain and neuroticism. To our knowledge, no previous studies have specifically examined the epidemiological relationships between neuroticism and hip pain or knee pain. Our findings suggest that hip pain and knee pain may belong to a separate pain group and should be considered separately when designing studies of the genetic relationships between pain and psychiatric disorders.

This paper also reported the genetic correlations among pain phenotypes in different body sites. Many pain phenotypes have positive and significant genetic correlations with each other indicating the common genetic mechanisms behind different pain phenotypes. This common mechanism is less likely to be represented by a few genes with large effects, but to reflect many genetic variants with smaller effects. It is biologically plausible for back pain and neck or shoulder pain to demonstrate the largest genetic correlation $(\mathrm{rg}=0.83)$ since causal genetic factors could have plausible detrimental effects across the whole spine. It is worth mentioning that neck, shoulder and back pain can be difficult to locate precisely, and can extend from one body area to the other; therefore phenotype and sample overlaps might also contribute to a certain extent. For other genetic correlations such as that between hip pain and pain all over the body ( $\mathrm{rg}=0.81)$, the reason is less apparent and merits further research. It is challenging to say that a specific pain site phenotype is mainly caused by musculoskeletal reasons, vascular issues or neurological factors. For example, vascular issues play a more important role than neurological factors in migraine, while considering multiple headache types as a whole, neurological factors take the leading role [40, 41]. Back pain is strongly associated with musculoskeletal causes, while neurological factors also contribute a part [42]. Interestingly, as we can see from the genetic correlation results, different pain phenotypes have shared genetic correlations. This means it might not be appropriate to include controls that have pain in other sites as this might cause false-negative results, especially for highly and genetically correlated pain phenotypes. We used self-reported migraine GWAS data from 23 andMe, Inc. as a further pain trait to calculate its genetic correlations with the UK Biobank pain phenotypes [40]. As the samples from 23andMe were from the USA, there was 
likely to be no sample overlaps with UK Biobank. The results supported our main findings in that there were shared genetic components between pain phenotypes across these two cohorts (Supplementary Fig. 1). We also performed a Mendelian randomisation (MR) analysis using headache as an exposure and psychiatric traits as outcomes to check if the genetic predisposition to headache also has causal effects to psychiatric outcomes. In addition, we also checked whether these psychiatric outcomes have causal effects to headache. The MR results showed that headache does have casual effects on depressive symptoms and neuroticism, but shows no causal effects on major depressive disorder based on the tradition inverse variance weighted method [43]. Neuroticism also has causal effects to headache, while major depressive disorders and depressive symptoms do not (Supplementary Table 3). Finally, the shared genetic contribution to pain phenotypes was further supported by the Mendelian randomisation analysis, which found that a genetic predisposition to headache resulted in a greater likelihood of symptoms of depression and neuroticism (and vice versa with neuroticism). To our knowledge, there have been no previous MR studies examining causal effects between headache or migraine and depression or neuroticism. Further work in this area is required.

The highest narrow-sense heritability among all pain phenotypes in this study was 0.31 for pain all over the body. The heritabilities of all other pain phenotypes were moderate. The narrow-sense heritability does not take gene-gene interactions, gene-environment interactions, or the contribution from rare variants into account, and is therefore likely to be an under estimate of the true heritability. This is the first report of the heritabilities for facial pain $\left(h^{2}=0.24\right)$, stomach or abdominal pain $\left(h^{2}=0.14\right)$, to the best of our knowledge, and suggests important genetic contributions to chronic pain at all body sites.

Arguably the greatest strength of the current study was the large size of the UK Biobank sample. Large sample sizes will allow researchers to overcome the potential issues of small sample sizes and limited study power (such as sample heterogeneity and sample selection biases) to cut through the statistical noise. This provided the largest single sample size for many of the pain phenotypes studied here compared with previous GWAS studies of pain [5]. Nevertheless, potential limitations should also be taken into account. The phenotyping in UK Biobank was based on a single specific non-standard pain-related question. This means that all pain phenotypes were broadly defined and unfiltered by other potentially relevant information on the nature, duration or intensity of the pain. Similar limitations also apply to the psychological traits measured in UK Biobank and elsewhere [44].
Currently, a new and more detailed, validated pain-related questionnaire is being administered to participants in UK Biobank, and this will allow for more detailed and focused phenotyping for use in future analyses, again with very large sample sizes. These can and should further analysis of the important gender difference in the genetic contributions to pain traits.

In summary, we have identified significant and positive genetic correlations between multiple pain phenotypes and depression and neuroticism, suggesting that the known associations between these traits are partly due to shared genetic architecture. In contrast, we have suggested that the known epidemiological relationships between hip and knee pain and depression are not caused primarily by common genetic factors, prompting a search for other explanations. In addition, we have shown that many pain phenotypes are heritable and have positive and significant genetic correlations with each other. This indicates that common genetic risk factors confer liability to pain at many different sites across the body, suggesting shared risk factors and, potentially, disease mechanisms.

These findings contribute to the understanding of the genetic and biological mechanisms for individual pain phenotypes, depression and neuroticism. In addition, the findings also represent an early but important step towards the identification of causal associations between pain phenotypes and psychiatric disorders and identifying subgroup heterogeneity.

\section{Data availability}

All GWAS summary statistics can be downloaded from https://figshare.com/articles/fourpainphenotypes1/7699556 https://figshare.com/articles/fourpainphenotype2/7699583

Acknowledgements We would like to thank all participants of the UK Biobank cohort who have provided necessary genetic and phenotypic information. We would like to thank the research participants and employees of 23 andMe for making this work possible.

Funding This work was supported by the STRADL project [Wellcome Trust, grant number: 104036/Z/14/Z], and the Centre for Cognitive Ageing and Cognitive Epidemiology [Medical Research Council and Biotechnology and Biological Sciences Research Council, grant number: MR/K026992/1]. We are grateful for support from the Sackler Foundation. The funders had no role in study design, data collection, data analysis, interpretation, writing of the report.

\section{Compliance with ethical standards}

Conflict of interest The authors declare that they have no conflict of interest.

Publisher's note Springer Nature remains neutral with regard to jurisdictional claims in published maps and institutional affiliations. 
Open Access This article is licensed under a Creative Commons Attribution 4.0 International License, which permits use, sharing, adaptation, distribution and reproduction in any medium or format, as long as you give appropriate credit to the original author(s) and the source, provide a link to the Creative Commons license, and indicate if changes were made. The images or other third party material in this article are included in the article's Creative Commons license, unless indicated otherwise in a credit line to the material. If material is not included in the article's Creative Commons license and your intended use is not permitted by statutory regulation or exceeds the permitted use, you will need to obtain permission directly from the copyright holder. To view a copy of this license, visit http://creativecommons. org/licenses/by/4.0/.

\section{References}

1. GBD 2017 Disease and Injury Incidence and Prevalence Collaborators Global, regional, and national incidence, prevalence, and years lived with disability for 354 diseases and injuries for 195 countries and territories, 1990-2017: a systematic analysis for the Global Burden of Disease Study 2017. Lancet. 2018;392:1789-858.

2. Enright A, Goucke R. The global burden of pain: the tip of the iceberg? Anesth Analg. 2016;123:529-30.

3. Treede RD, Rief W, Barke A, Aziz Q, Bennett MI, Benoliel R, et al. A classification of chronic pain for ICD-11. Pain. 2015;156:1003-7.

4. Zorina-Lichtenwalter K, Meloto CB, Khoury S, Diatchenko L. Genetic predictors of human chronic pain conditions. Neuroscience. 2016;338:36-62.

5. Peters MJ, Broer L, Willemen HL, Eiriksdottir G, Hocking LJ, Holliday KL, et al. Genome-wide association study meta-analysis of chronic widespread pain: evidence for involvement of the 5p15.2 region. Ann Rheum Dis. 2013;72:427-36.

6. Dominick CH, Blyth FM, Nicholas MK. Unpacking the burden: understanding the relationships between chronic pain and comorbidity in the general population. Pain. 2012;153:293-304.

7. Kadimpati S, Zale EL, Hooten MW, Ditre JW, Warner DO. Associations between neuroticism and depression in relation to catastrophizing and pain-related anxiety in chronic pain patients. PLoS ONE. 2015;10:e126351.

8. Cuijpers P, Smit F, Penninx BW, de Graaf R, ten Have M, Beekman AT. Economic costs of neuroticism: a population-based study. Arch Gen Psychiatry. 2010;67:1086-93.

9. Samaan Z, Farmer A, Craddock N, Jones L, Korszun A, Owen M, et al. Migraine in recurrent depression: case-control study. Br J Psychiatry. 2009;194:350-4.

10. Korszun A. Facial pain, depression and stress-connections and directions. J Oral Pathol Med. 2002;31:615-9.

11. Blozik E, Laptinskaya D, Herrmann-Lingen C, Schaefer H, Kochen MM, Himmel W, et al. Depression and anxiety as major determinants of neck pain: a cross-sectional study in general practice. BMC Musculoskelet Disord. 2009;10:13.

12. Walter SA, Jones MP, Talley NJ, Kjellström L, Nyhlin H, Andreasson AN, et al. Abdominal pain is associated with anxiety and depression scores in a sample of the general adult population with no signs of organic gastrointestinal disease. Neurogastroenterol Motil. 2013;25:741-e576.

13. Tsuji T, Matsudaira K, Sato H, Vietri J. The impact of depression among chronic low back pain patients in Japan. BMC Musculoskelet Disord. 2016;17:447.

14. Gracely RH, Ceko M, Bushnell MC. Fibromyalgia and depression. Pain Res Treat. 2012;2012:486590.

15. Ashina S, Bendtsen L, Buse DC, Lyngberg AC, Lipton RB, Jensen R. Neuroticism, depression and pain perception in migraine and tension-type headache. Acta Neurol Scand. 2017;136:470-6.
16. Bru E, Mykletun RJ, Svebak S. Neuroticism, extraversion, anxiety and Type A behaviour as mediators of neck, shoulder and lower back pain in female hospital staff. Pers Individ Differ. 1993;15:485-92.

17. Lefebvre JC, Keefe FJ. The effect of neuroticism on the recall of persistent low-back pain and perceived activity interference. J Pain. 2013;14:948-56.

18. Malin K, Littlejohn GO. Neuroticism in young women with fibromyalgia links to key clinical features. Pain Res Treat. 2012; 2012:730741.

19. McIntosh AM, Hall LS, Zeng Y, Adams MJ, Gibson J, Wigmore E, et al. Genetic and environmental risk for chronic pain and the contribution of risk variants for major depressive disorder: a family-based mixed-model analysis. PLoS Med. 2016;13:e1002090.

20. van Hecke O, Hocking LJ, Torrance N, Campbell A, Padmanabhan $\mathrm{S}$, Porteous DJ, et al. Chronic pain, depression and cardiovascular disease linked through a shared genetic predisposition: analysis of a family-based cohort and twin study. PLoS ONE. 2017;12:e0170653.

21. Pingault JB, O'Reilly PF, Schoeler T, Ploubidis GB, Rijsdijk F, Dudbridge F. Using genetic data to strengthen causal inference in observational research. Nat Rev Genet. 2018;19:566-80.

22. Stam AH, de Vries B, Janssens AC, Vanmolkot KR, Aulchenko YS, Henneman P, et al. Shared genetic factors in migraine and depression: evidence from a genetic isolate. Neurology. 2010;74:288-94.

23. Ligthart L, Boomsma DI. Causes of comorbidity: pleiotropy or causality? Shared genetic and environmental influences on migraine and neuroticism. Twin Res Hum Genet. 2012;15:158-65.

24. Pinheiro MB, Morosoli JJ, Colodro-Conde L, Ferreira PH, Ordoñana JR. Genetic and environmental influences to low back pain and symptoms of depression and anxiety: a population-based twin study. J Psychosom Res. 2018;105:92-8.

25. Bulik-Sullivan B, Finucane HK, Anttila V, Gusev A, Day FR, Loh $\mathrm{PR}$, et al. An atlas of genetic correlations across human diseases and traits. Nat Genet. 2015;47:1236-41.

26. Bycroft C, Freeman C, Petkova D, Band G, Elliott LT, Sharp K, et al. The UK Biobank resource with deep phenotyping and genomic data. Nature. 2018;562:203-9.

27. Okbay A, Baselmans BM, Neve JE, Turley P, Nivard MG, Fontana MA, et al. Genetic variants associated with subjective wellbeing, depressive symptoms, and neuroticism identified through genome-wide analyses. Nat Genet. 2016;48:624-33.

28. van den Berg SM, de Moor MH, McGue M, Pettersson E, Terracciano A, Verweij KJ, et al. Harmonization of neuroticism and extraversion phenotypes across inventories and cohorts in the genetics of personality consortium: an application of item response theory. Behav Genet. 2014;44:295-313.

29. Major Depressive Disorder Working Group of the Psychiatric GWAS Consortium, Ripke S, Wray NR, Lewis CM, Hamilton SP, Weissman MM, et al. A mega-analysis of genome-wide association studies for major depressive disorder. Mol Psychiatry. 2013;18:497-511.

30. Yang J, Lee SH, Goddard ME, Visscher PM. GCTA: a tool for genome-wide complex trait analysis. Am J Hum Genet. 2011;88: 76-82.

31. Han B, Pouget JG, Slowikowski K, Stahl E, Lee CH, Diogo D, et al. A method to decipher pleiotropy by detecting underlying heterogeneity driven by hidden subgroups applied to autoimmune and neuropsychiatric diseases. Nat Genet. 2016;48:803-10.

32. Bair MJ, Robinson RL, Katon W, Kroenke K. Depression and pain comorbidity: a literature review. Arch Intern Med. 2003;163: 2433-45.

33. Gasperi M, Herbert M, Schur E, Buchwald D, Afari N. Genetic and environmental influences on sleep, pain, and depression symptoms in a community sample of twins. Psychosom Med. 2017;79:646-54. 
34. Descalzi G, Mitsi V, Purushothaman I, Gaspari S, Avrampou K, Loh YE, et al. Neuropathic pain promotes adaptive changes in gene expression in brain networks involved in stress and depression. Sci Signal. 2017;10:eaaj1549. pii

35. Hashmi JA, Baliki MN, Huang L, Baria AT, Torbey S, Hermann $\mathrm{KM}$, et al. Shape shifting pain: chronification of back pain shifts brain representation from nociceptive to emotional circuits. Brain. 2013;136:2751-68.

36. Gandhi R, Zywiel MG, Mahomed NN, Perruccio AV. Depression and the overall burden of painful joints: an examination among individuals undergoing hip and knee replacement for osteoarthritis. Arthritis. 2015;2015:327161.

37. Sugai K, Takeda-Imai F, Michikawa T, Nakamura T, Takebayashi T, Nishiwaki Y. Association between knee pain, impaired function, and development of depressive symptoms. J Am Geriatr Soc. 2018;66:570-6.

38. Phyomaung PP, Dubowitz J, Cicuttini FM, Fernando S, Wluka AE, Raaijmaakers $\mathrm{P}$, et al. Are depression, anxiety and poor mental health risk factors for knee pain? A systematic review. BMC Musculoskelet Disord. 2014;15:10.
39. Payne LA, Seidman LC, Lung KC, Zeltzer LK, Tsao JC. Relationship of neuroticism and laboratory pain in healthy children: does anxiety sensitivity play a role? Pain. 2013;154:103-9.

40. Gormley P, Anttila V, Winsvold BS, Palta P, Esko T, Pers TH, et al. Meta-analysis of 375,000 individuals identifies 38 susceptibility loci for migraine. Nat Genet. 2016;48:856-66.

41. Meng W, Adams MJ, Hebert HL, Deary IJ, McIntosh AM, Smith $\mathrm{BH}$. A genome-wide association study finds genetic associations with broadly-defined headache in UK Biobank $(\mathrm{N}=223,773)$. EBioMedicine. 2018;28:180-6.

42. Suri P, Palmer MR, Tsepilov YA, Freidin MB, Boer CG, Yau MS, et al. Genome-wide meta-analysis of 158,000 individuals of European ancestry identifies three loci associated with chronic back pain. PLoS Genet. 2018;14:e1007601.

43. De Silva NMG, Borges MC, Hingorani AD, Engmann J, Shah T, Zhang $\mathrm{X}$, et al. Liver function and risk of Type 2 diabetes: bidirectional Mendelian randomization study. Diabetes. 2019;68:1681-91.

44. Ormel J, Jeronimus BF, Kotov R, Riese H, Bos EH, Hankin B, et al. Neuroticism and common mental disorders: meaning and utility of a complex relationship. Clin Psychol Rev. 2013;33:686-97. 